\title{
Fuzzy arithmetic in economic research
}

\author{
Kateryna Gorbatiuk ${ }^{1 *}$, Tetiana Zavhorodnia ${ }^{1}$, Oksana Proskurovych $^{1}$, and Olha Mantalyuk ${ }^{1}$ \\ ${ }^{1}$ Khmelnytskyi National University, 11 Instytutska Str., Khmelnytskyi, 29016, Ukraine
}

\begin{abstract}
Many tasks in economic research are based on arithmetic calculations of indicators that reflect the state of economic development. The general incompleteness of publicly available data, designed to solve such problems, has led to the emergence of numerous decision support systems based on fuzzy arithmetic. The article presents a study on the approach aimed at integrating fuzzy information about economic indicators into economic models. The definition of arithmetic operations on fuzzy values is given, and the methods of obtaining the resulting fuzzy indicators with the help of some software tools are considered. Analytical and numerical methods of obtaining the resulting indicators in the form of fuzzy numbers are described and analyzed. A direct calculation algorithm for all arithmetic operations is proposed, utilized, and used for the evaluation of resulting indicators. Also, analytical and numerical methods for obtaining fuzzy results are considered in the article, and some of them are proposed for utilization. On the example of economic indicators used in the labor rationing, the results of an evaluation of some indicators for a technological operation in the form of fuzzy numbers were obtained by different methods and compared. The practical recommendations, given in the article, on the use of fuzzy arithmetic in decision support systems outline the directions of further research.
\end{abstract}

\section{Introduction}

To make an effective management decision under the conditions of uncertainty and risk, it is often required to take into account incomplete information about the indicators that affect the efficiency of processes in various sectors of the economy. So, the tasks of assessing economic indicators are often performed taking into account the uncertainty. One of the ways to describe the incompleteness of information in indicators of different nature is to formalize the uncertainty using the fuzzy set theory approach.

The presentation of economic indicators in the form of fuzzy numbers opens up ample opportunities for taking into account uncertainty and using all available information in calculations without significant losses due to assumptions about the determinism and clarity of specific values. Working with economic indicators presented as fuzzy values requires the use of appropriate calculation methods, in particular, using the software. This will automate the fuzzy assessment of indicators obtained by establishing the relationship between factors described as fuzzy numbers.

The fuzzy set theory, proposed in 1965 by L. A. Zadeh $[1,2]$ is becoming more and more widespread in the practice of modeling uncertainties in indicators of various natures. In particular, the methods and basic concepts of the fuzzy set theory have found their application in economic research tasks.

Thus, if we move away from the idea of considering the economic indicators as random values with unknown probabilistic characteristics and move on to the concepts

*e-mail: ecvgor@ukr.net of the fuzzy set theory, we can obtain full advantage of the fuzzy set theory technique over the technique of probability theory, which does not provide a suitable way to answer many practical questions.

At the same time, the similarity in the concepts of fuzziness and randomness allows us to draw correspondences between the membership functions in the fuzzy set theory and likelihood characteristics in probability theory.

In many works devoted to the mathematical modeling of various economic processes, it is noted that the practice of modeling economic indicators with only average characteristics is not always acceptable. Most indicators that take into account only the average values should also be described with a margin of error estimate. According to the authors of this article, most of the economic indicators should be described using fuzzy values (fuzzy numbers). Moreover, the construction of their membership functions can be based both on the use of expert information and on a statistical analysis of the distributions of the studied indicators.

The main purposes of this study are: considering the existing fuzzy arithmetic methods; applying fuzzy arithmetic methods to the problems of fuzzy estimation of economic indicators on the example of calculating indicators in the field of labor rationing; comparing the results of estimation obtained by different methods, in particular, implemented in the programming language $\mathrm{R}$; providing recommendations for further use of fuzzy arithmetic for assessing economic indicators taking into account an uncertainty of fuzzy nature.

There are six sections in this article. The first one contains the description of the background of this research. 
The second section presents an overview of the scientific sources on the topic of research. The theoretical details of the proposed methods are explained in the third section. Then, the stages of the study and their main results are shown in the fourth and fifth parts of this work. The last part of the article contains several conclusions based on the research results and covers a discussion on further research directions.

\section{Literature review}

Since 1965 [1], the methods and basic concepts of fuzzy set theory have become widespread in the practice of solving economic problems. The introduction of the fuzzy sets' concept, classes with inaccurate boundaries described by membership functions, provided a basis for the development of a more flexible approach to the analysis of judgments and modeling of complex systems, whose behavior is described by linguistic rather than numerical variables.

Working with fuzzy indicators requires the use of appropriate theoretically sound calculation methods, which is impossible without the use of modern computing tools. The basic concepts of fuzzy arithmetic are represented in different scientific works all over the world. In particular, the articles [3-9] contain the description and theoretical reasoning of the most widely used methods of fuzzy calculations. Some of the studies [10-13] are provided to improve the basic ideas by the new computational approaches. These works are only a small part of the total number of works in this area.

Recently, many software products have been created for fuzzy calculations, which allow taking into account the need to perform arithmetic operations on fuzzy values. One such software is the R programming language, which contains the corresponding function libraries, which are constantly supplemented by implementations of new methods. Work with these methods is described in detail in the relevant literature [14-16], which simplifies their practical application for fuzzy calculations.

Also, some implementations of fuzzy arithmetic are presented in the studies [17-20] related to several economic tasks. In [17] is proposed a method based on fuzzy arithmetic, designed to estimate the possible range of flow rates (and levels) of water, to make a forecast, based on possible rainfalls provided by the uncertainty model. The paper [18] outlines a computational method for decreasing the imperfection of the fuzzy arithmetic models output, where was suggested an approach aiming at integrating into the arithmetic models fuzzy information still not taken into account: the imprecise knowledge about the interaction potentially existing between two variables when performing an arithmetic operation (addition, subtraction, division or product). The article [19] represents a model of the power plant as a multivariable system using fuzzy arithmetic based on the Transformation Method. Finally, in cash flow analysis [20], instead of single-valued estimation, each cost data value is proposed to be designated as a trapezoidal fuzzy set number.

\section{Research methodology}

Fuzzy set theory tools allow to intuitively display inaccurate or fuzzy data. Fuzzy numbers form a subclass of fuzzy sets built on subsets of real numbers. By definition, a fuzzy set $A$ with a membership function $\mu_{A}: R \rightarrow[0,1]$ is a fuzzy number if it has at least the following properties:

1) it is a normalized fuzzy set, i.e. $\mu_{A}\left(x_{0}\right)=1$ for a certain value $x_{0} \in R$;

2) the membership function is convex, i.e. it is true that $\mu_{A}\left(\lambda x_{1}+(1-\lambda) x_{2}\right) \geq \mu_{A}\left(x_{1}\right) \wedge \mu_{A}\left(x_{2}\right)$ for any $x_{1}, x_{2} \in R$ and $\lambda \in[0,1]$;

3) the carrier set (universe of discourse) of the fuzzy set $A$ is limited.

The specific types of membership functions are determined based on various additional assumptions about the properties of these functions (symmetry, monotonicity, continuity of the first derivative, etc.) taking into account the specifics of the existing uncertainty and the real situation [4].

In particular, fuzzy numbers with a triangular membership function $\mu(t)$ are called triangular fuzzy numbers, and they are denoted by $\bar{t}=\left(t_{\min } / t_{c} / t_{\max }\right)$, where $t_{\min }, t_{\max }, t_{c}-$ the minimum, maximum value, and some measure of the central value (mean, mode, median, etc.) of a particular parameter, respectively. The membership function of this triangular fuzzy number is:

$$
\mu(t)=\left\{\begin{array}{l}
\frac{t-t_{\min }}{t_{c}-t_{\min }}, \text { if } t_{\min } \leq t \leq t_{c}, \\
\frac{t-t_{\max }}{t_{c}-t_{\max }}, \text { if } t_{c} \leq t \leq t_{\max } .
\end{array}\right.
$$

To solve the problem of inflexibility and incompleteness of using single-valued estimates of economic indicators, fuzzy numbers can be employed to deal with the uncertainty in economic analysis.

There is no doubt about the need to automate calculations on determining the results of arithmetic operations on fuzzy numbers. Also, the implementation of the fuzzy arithmetic methods requires using of computer technologies, which can allow developing the corresponding algorithms. Some of the algorithms were developed by the authors using VBA in Excel, and utilized for fuzzy arithmetic calculations of economic indicators in the field of labor rationing.

Nowadays, the design of fuzzy systems is assisted by the general-purpose fuzzy systems software (FSS) that has been actively developed during recent years [15]. Most of this FSS is released to free and open-source libraries and toolboxes with the aim of being reusable and easy to integrate with software developed for specific application purposes [15].

One of such general-purpose fuzzy systems software is an $\mathrm{R}$ programming language (with RStudio environment). $\mathrm{R}$ is a free, open-source language that is already widely used in various fields (from computer science to social sciences) [14]. At present, the $\mathrm{R}$ programming language is gaining importance as a non-commercial solution that 
is competitive with Matlab [15]. Thus, the provision of freely available software for the use of non-classical fuzzy systems within the $\mathrm{R}$ packages allows practitioners and researchers from a wide range of fields to use in their research the developments of the entire research community [14].

Fuzzy numbers, which play an important role in many practical applications, have a software implementation in the R package. FuzzyNumbers is an open-source package for the $\mathrm{R}$ programming language, which deals with all major operating systems, i.e. Windows, Linux, and macOS [16]. FuzzyNumbers is designed for easy and efficient use of fuzzy numbers and various fuzzy operations.

A fuzzy number $A$ can be specified by giving its carrier set (universe of discourse), or left/right side functions, or lower/upper limits of $\alpha$-cuts [16]. In practice, most algorithms that work with fuzzy numbers involve the use of at least the latter, i.e. $\alpha$-cuts.

A fuzzy number given by side functions (LR fuzzy number) has the following form of membership function [16]:

$$
\mu_{A}(x)=\left\{\begin{array}{l}
0, \text { if } x<a_{1}, \\
\text { left }\left(\frac{x-a_{1}}{a_{2}-a_{1}}\right), \text { if } a_{1} \leq x<a_{2}, \\
1, \text { if } a_{2} \leq x \leq a_{3} \\
\text { right }\left(\frac{x-a_{3}}{a_{4}-a_{3}}\right), \text { if } a_{3}<x \leq a_{4}, \\
0, \text { if } x>a_{4} .
\end{array}\right.
$$

where $a_{1}, a_{2}, a_{3}, a_{4} \in R, a_{1} \leq a_{2} \leq a_{3} \leq a_{4}$, left - is a non-decreasing function (left generator $A$ ), right - is a non-increasing function (right generator $A$ ).

For example, a fuzzy number $A$ with linear side functions (trapezoidal number) can be specified in $\mathrm{R}$ as: "A->FuzzyNumber $(1,2,4$, 7, left = function $(\mathrm{x}) \mathrm{x}$, right $=$ function $(\mathrm{x}) 1-\mathrm{x})$ ".

To graphically represent a given fuzzy number we can use the command: "plot (A)" (figure 1).

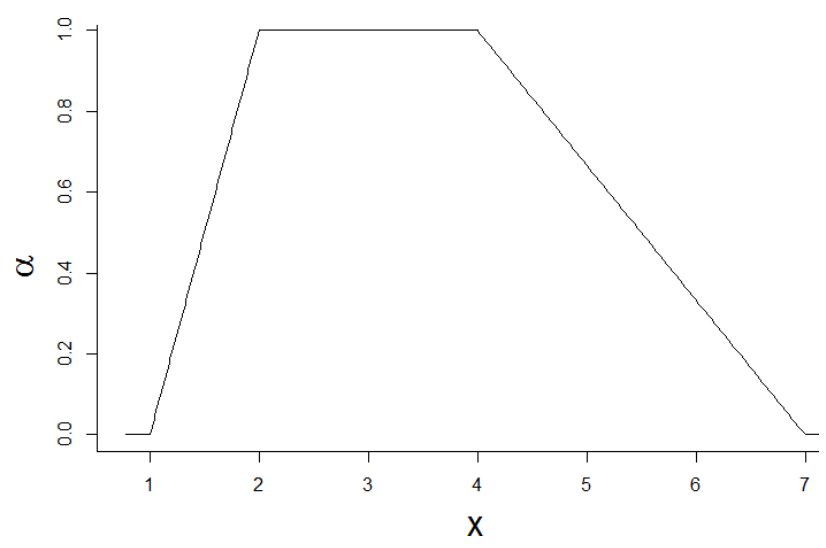

Figure 1. Membership function of a trapezoidal fuzzy number

\footnotetext{
A similar trapezoidal fuzzy number can also be specified using the function: "TrapezoidalFuzzyNumber(1,2,4,7)].

So, to specify such a number, we must provide four parameters: $a_{1}, a_{2}, a_{3}, a_{4} \in R$.
}

Also, the FuzzyNumbers package contains the methods for performing arithmetic operations on different types of fuzzy numbers. But, while addition and subtraction are fairly simple operations, multiplication and division, unfortunately, remain unclosed and are more complex [8], and do not always have a solution.

\section{Case study: fuzzy arithmetic methods in calculating the economic indicators}

\subsection{Direct and inverse methods of performing arithmetic operations on fuzzy numbers}

If we are talking about the economic indicators in different areas of research, the shape and values of membership functions of fuzzy numbers can be obtained using expert information, as well as using probabilistic methods based on certain similarities between the concepts of fuzziness and probability. Without the ability to accurately determine the type of distribution, from which the samples are taken, we only can deal with the distribution frequency characteristics.

We propose the method of fuzzy representation of economic indicators based on statistical data. Thus, based on statistical data of selected economic indicators, appropriate distribution histograms or frequency polygons can be constructed with the number of intervals $m$ in the range from 7 to 12 according to the recommendations for histogram building [21]. Each value of the membership function $\mu$ of the corresponding indicator is calculated for the middle of each interval as a relative frequency divided by the maximum value of all frequencies.

Then, it is possible to obtain a fuzzy number $A=\left\langle\mu_{A} ;[a ; b]\right\rangle$ with a discretized membership function $\mu_{A}$ given on the real interval $[a ; b]$, which is divided into $m$ equal parts: $[a ; b]=\left[b_{0} ; b_{1}\right] \cup\left[b_{1} ; b_{2}\right] \cup \ldots\left[b_{m-1} ; b_{m}\right]$, where $b_{0}=a, b_{m}=b$. Each value $\mu_{A}\left(b_{i}\right)$ of the membership function corresponds to the value $b_{i}, i=\overline{0, m}$. Therefore, $\mu_{A}$ is a point membership function specified on the real interval $[a ; b]$.

In probability theory, there are no general definitions or methods for determining the parameters of the distribution of an indicator, which is the result of performing arithmetic operations on random variables. And if we need to form our opinion on the shape of the indicator's resulting distribution, we need to build modeling (simulating) algorithms.

On the contrary, in fuzzy set theory, there are several methods designed to obtain the results of arithmetic operations on fuzzy numbers. Among them, there are analytical and numerical methods, as well as direct and inverse methods. Numerical methods are more universal and convenient than the analytical methods for use in practical problems of automated calculations.

The fuzzy extension principle, introduced by Zadeh in $[1,2]$, is the basic tool for fuzzy arithmetic. It extends functions of real numbers to functions of fuzzy numbers and it allows the extension of arithmetic operations to fuzzy arguments [4]. According to the extension principle, the following definition of a membership function for 
a fuzzy number, which is the result of an arithmetic operation on two fuzzy numbers $A$ and $B$, is:

$$
\mu_{A \circ B}(z)=\sup _{U}\left\{\mu_{A}(x) \wedge \mu_{B}(y)\right\}
$$

where $U=\{(x, y) \in \sigma(A \times B) \mid x \circ y=z\},\{\circ\}-$ is an arithmetic operation from the set $\{+,-, *, /\}, \sigma(A \times B)$ is the Cartesian product of the partitions of the fuzzy set carriers $A$ and $B$.

If we use this definition directly, and try to calculate the resulting membership function values as required in formula (3), then we obtain the method for determining the result of arithmetic operations on fuzzy numbers called a direct method.

In addition to direct methods, there are several inverse methods for solving the problem, based on so-called $\alpha$ cuts representation. By the $\alpha$-cuts approach, it is possible to define a parametric representation of fuzzy numbers that allows a large variety of possible shapes, and is very simple to implement [4]. Both analytical and numerical methods have been developed in the theory of fuzzy sets, in particular, there are matrix methods. One of them, described in [9], allows finding a solution for any arithmetic operation from the set $\{+,-, *, /\}$, provided that the membership functions of the numbers, combined by these operations, are convex. And also, the division operation requires compliance with the condition: $0 \notin[c ; d]$, where $[c ; d]$ - is the carrier of a fuzzy number representing the divisor.

The difference between the inverse methods and the direct ones is in the fact, that the discretization of the membership function in inverse methods is not based on the parameter $X$, but on the value of $\alpha \in[0 ; 1]$, i.e. $\alpha$-level sets ( $\alpha$-cuts) are constructed for the values of $\alpha$ (for example, $\alpha=0.2,0.4,0.6,0.8$ and 1). But in this case, there is an additional issue with determining of the value $x_{i}$ for each level $\alpha$. That is due to the fact that the membership function is built by frequencies based on the uniform partition of the parameter $X$, which does not guarantee the presence of exact values of $\alpha$ at the obtained partition points.

We assume, that if the value is given by the discretized membership function $\mu$ by the parameter $X$, then in the interval between the nodes of the partition, $\mu$ is approximated by a linear function $a x+b$, which parameters are calculated by the formulas:

$$
a=\frac{\mu\left(x_{1}\right)-\mu\left(x_{2}\right)}{x_{1}-x_{2}} ; b=\frac{\mu\left(x_{2}\right) x_{1}-\mu\left(x_{1}\right) x_{2}}{x_{1}-x_{2}}
$$

where $\left(x_{1}, y_{1}\right),\left(x_{2}, y_{2}\right)$ - the partition nodes.

Then, the value $x_{i}$ for a given $\alpha$-level could be calculated by the formula: $x_{i}=\frac{\alpha-b}{a}$, having determined in advance on which section, relative to the nodes, the value of $\alpha$ is.

Thus, having the membership function discretized by $\alpha$-levels, we will be able to apply the matrix method [9] for finding the results of arithmetic operations on fuzzy numbers. To do this, we construct a matrix of dimension $18 \times 18$ (there are nine values of $\alpha$-levels $=0.2,0.4,0.6$, $0.8,1.0,0.8,0.6,0.4,0.2$ for each fuzzy number). Even values of matrix indices both in rows and columns will correspond to the values of arithmetic operation results (performed on the particular values of the carriers of $A$ and $B$ ). Odd indexes will indicate the level of the corresponding membership function, which is the result of the operation:

$$
\mu_{c}\left(x_{i j}\right)=\min \left(\mu\left(x_{A i}\right) ; \mu\left(x_{B j}\right)\right) .
$$

So, the results of the operation $x_{i j}=x_{A i} \circ x_{B j}$ are placed in the cells of the matrix with even indices $i$ and $j$. All other cells of the matrix remain unfilled.

Studies show that the solution for the resulting function is located depending on the type of operation on one of the matrix diagonals: for addition and multiplication on the diagonal that runs from the upper left corner to the lower right corner, and for subtraction and division - on another diagonal.

Then, we carry out the numerical experiment to find the result of the multiplication of two fuzzy numbers $A$ and $B$ by the method of direct application of the formula (3), and also by the method of a full scan of frequency characteristics with subsequent multiplication of corresponding data values.

The membership functions of fuzzy numbers are often based on expert information. However, there are also probabilistic methods for constructing membership functions based on the similarity of the concepts of fuzziness and probability.

Suppose, that there is statistical data on some economic indicators $A$ and $B$. According to the obtained samples, the corresponding frequency histograms are constructed using the number of intervals in the range defined as recommended by Sturges' Rule for building histograms [21]. But, in our study, we have chosen the number of bins quite larger, to have more data points for calculations, and we set $m=19$.

To obtain an unambiguous correspondence between the values of $A$ and $B$ to the frequencies, we replace the frequency histogram with another graphical characteristic used in probability theory and mathematical statistics - a frequency polygon. The values of the fuzzy number carriers for $A$ and $B$ and the corresponding frequencies $N$ are represented in table 1 .

So, we construct the membership function for each number $A$ and $B$ based on the available frequency polygons. To do this, we normalize the values by dividing the current frequencies by the maximum frequency value $n_{\max }$. Thus, we obtain two fuzzy numbers: $A=\left\langle\mu_{A} ;[a ; b]\right\rangle$ and $B=\left\langle\mu_{B} ;[c ; d]\right\rangle$, where $\mu_{A}, \mu_{B}-$ are the discretized membership functions given on the carriers $[a ; b]$ and $[c ; d]$ (real intervals) respectively.

Moreover, the real intervals $[a ; b]$ and $[c ; d]$ are divided into $m$ equal parts: $[a ; b]=\left[b_{0} ; b_{1}\right] \cup\left[b_{1} ; b_{2}\right] \cup$ $\ldots\left[b_{m-1} ; b_{m}\right]$, where $b_{0}=a, b_{m}=b$; and $[c ; d]=\left[d_{0} ; d_{1}\right] \cup$ $\left[d_{1} ; d_{2}\right] \cup \ldots\left[d_{m-1} ; d_{m}\right]$, where $d_{0}=c, d_{m}=d$.

Thus, two point membership functions are set on the intervals $[a ; b]$ and $[c ; d]$ by the values $b_{i}, i=\overline{0, m}$ and $d_{i}, i=\overline{0, m}$, and corresponding values of the membership functions $\mu_{A}$ and $\mu_{B}$.

Now, we need to construct the membership function of the fuzzy number $A B$ directly using the definition (3). 
Table 1. Membership functions for $A, B$ and $A B$

\begin{tabular}{cccccccc}
\hline$A$ & $N$ & $\mu_{A}$ & $B$ & $N$ & $\mu_{B}$ & $A B$ & $\mu_{a b}$ \\
\hline 0.05 & 1 & 0.03 & 0.37 & 1 & 0.02 & 0.02 & 0.07 \\
0.07 & 2 & 0.07 & 0.39 & 1 & 0.03 & 0.03 & 0.32 \\
0.10 & 11 & 0.32 & 0.41 & 3 & 0.08 & 0.05 & 0.54 \\
0.12 & 17 & 0.49 & 0.42 & 6 & 0.17 & 0.07 & 0.73 \\
0.15 & 23 & 0.67 & 0.44 & 8 & 0.24 & 0.09 & 0.89 \\
0.17 & 25 & 0.73 & 0.46 & 19 & 0.54 & 0.10 & 1.00 \\
0.20 & 34 & 1.00 & 0.48 & 17 & 0.49 & 0.12 & 0.86 \\
0.22 & 33 & 0.96 & 0.50 & 26 & 0.77 & 0.14 & 0.76 \\
0.25 & 29 & 0.86 & 0.51 & 30 & 0.89 & 0.15 & 0.59 \\
0.27 & 23 & 0.67 & 0.53 & 34 & 1.00 & 0.17 & 0.55 \\
0.30 & 19 & 0.55 & 0.55 & 28 & 0.82 & 0.19 & 0.41 \\
0.32 & 14 & 0.41 & 0.57 & 26 & 0.77 & 0.21 & 0.25 \\
0.35 & 9 & 0.25 & 0.59 & 20 & 0.59 & 0.22 & 0.14 \\
0.37 & 4 & 0.12 & 0.61 & 14 & 0.41 & 0.24 & 0.14 \\
0.39 & 5 & 0.14 & 0.62 & 9 & 0.27 & 0.26 & 0.07 \\
0.42 & 2 & 0.07 & 0.64 & 5 & 0.15 & 0.27 & 0.05 \\
0.44 & 2 & 0.04 & 0.66 & 2 & 0.05 & 0.29 & 0.04 \\
0.47 & 1 & 0.02 & 0.68 & 2 & 0.04 & 0.31 & 0.02 \\
0.49 & 1 & 0.02 & 0.70 & 1 & 0.02 & 0.33 & 0.01 \\
\hline
\end{tabular}

To do this, for each element of the Cartesian product of the partitions of the fuzzy set carriers $A$ and $B$, we calculate the value $b_{i} \cdot d_{j}$ for all $i, j=\overline{0, m}$. Therefore, we obtain a matrix of dimension $(m+1) \times(m+1)$. For the obtained range of values, we find the minimum and maximum elements to determine the carrier for the resulting fuzzy number $[w ; z]$. Then, we divide the obtained real interval by the points $z_{i}$ into $m$ equal parts $(i=\overline{0, m}):[w ; z]=\left[z_{0} ; z_{1}\right] \cup\left[z_{1} ; z_{2}\right] \cup$ $\ldots\left[z_{m-1} ; z_{m}\right]$, where $z_{0}=w, z_{m}=z$.

For each part, we determine its middle point:

$$
z_{k}^{*}=\frac{z_{k}+z_{k+1}}{2}
$$

where $k=\overline{0, m-1}$.

From the set of Cartesian product elements, we select those pairs $\left(b_{i}, d_{j}\right)$, for which $b_{i} \cdot d_{j} \in\left[z_{k} ; z_{k+1}\right]$. For each such pair $\left(b_{i}, d_{j}\right)$, we determine $\mu_{C}\left(b_{i}, d_{j}\right)=\mu_{A}\left(b_{i}\right) \wedge \mu_{B}\left(d_{j}\right)$ (minimum of $\mu_{A}$ and $\mu_{B}$ ), and set the corresponding $\mu_{c}\left(z_{k}^{*}\right)$ to the maximum of $\mu_{C}\left(b_{i}, d_{j}\right)$. The process is repeated for each interval $\left[z_{k} ; z_{k+1}\right]$, where $k=\overline{0, m-1}$.

So, using the developed algorithm it is possible to obtain a discrete membership function as the result of multiplying of two fuzzy numbers (table 1).

For comparison, we can simulate the distribution of the values $A B$ directly using the data of the frequency characteristics of $A$ and $B$. That is, we "unfold" the frequency distribution into a set of values according to the frequency of each value. For the obtained samples we perform the operation of pairwise multiplication of all possible sample values. As a result, we obtain a set of 62500 values, which can be used to construct a frequency polygon, and consequently determine the membership function $\mu_{C}$.

Then, we compare the fuzzy numbers obtained by two different methods (figure 2).

The graphs show the absence of significant differences in the constructed membership functions. But it is quite

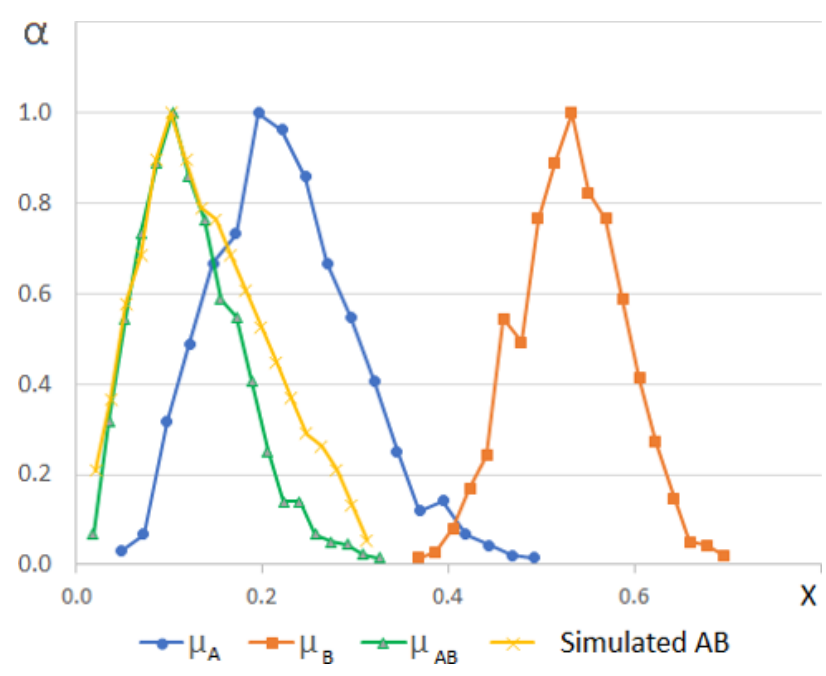

Figure 2. Membership functions of original and resulting fuzzy numbers

obvious that the amount of calculations in the direct "multiplication" of the samples is always significantly larger.

\subsection{Using fuzzy arithmetic in labor rationing tasks}

The proposed methods can be tested on examples of assessing indicators used in labor rationing. Consider the time of maintenance of workplace $\left(T_{\text {tech }}\right)$, which is calculated by the formula:

$$
T_{\text {tech }}=\frac{T_{n}}{T_{f}} \cdot T_{m},
$$

where $T_{f}$ - the time of equipment failure at the workplace; $T_{m}$ - machine operation time;

$T_{n}$ - the rate of time (or complexity) for the elimination of the failure, equal to the downtime of the workplace.

In this case, to describe the characteristics required for the calculations, we will use triangular fuzzy numbers.

So, let $\overline{T_{n}}=(10 / 15 / 20)(\min ),. \overline{T_{f}}=(200 / 250 / 300)$ (min.). The value $T_{m}$ is given as a crisp real number, and it is equal to 20 (min.).

Applying the division operation to the fuzzy numbers $T_{n}$ and $T_{f}$, we obtain the result (after multiplying by $T_{m}$ ): $\overline{T_{\text {tech }}}=(0.625 / 1.132 / 1.905)$. The table 2 is filled with intermediate and resulting calculations.

Table 2. Calculation of workplace maintenance time

\begin{tabular}{ccccc}
\hline$\alpha$ & $\overline{T_{n}}$ & $\overline{T_{f}}$ & $\overline{T_{n}} / \overline{T_{f}}$ & $\bar{T}_{\text {tech }}$ \\
\hline 0.00 & 10.00 & 200.00 & 0.031 & 0.625 \\
0.33 & 11.67 & 216.67 & 0.039 & 0.773 \\
0.67 & 13.33 & 233.33 & 0.047 & 0.941 \\
1.00 & 15.00 & 250.00 & 0.057 & 1.132 \\
0.67 & 16.67 & 266.67 & 0.068 & 1.351 \\
0.33 & 18.33 & 283.33 & 0.080 & 1.606 \\
0.00 & 20.00 & 300.00 & 0.095 & 1.905 \\
\hline
\end{tabular}

A graphic representation of the obtained membership function of the workplace maintenance time is shown in figure 3 . 


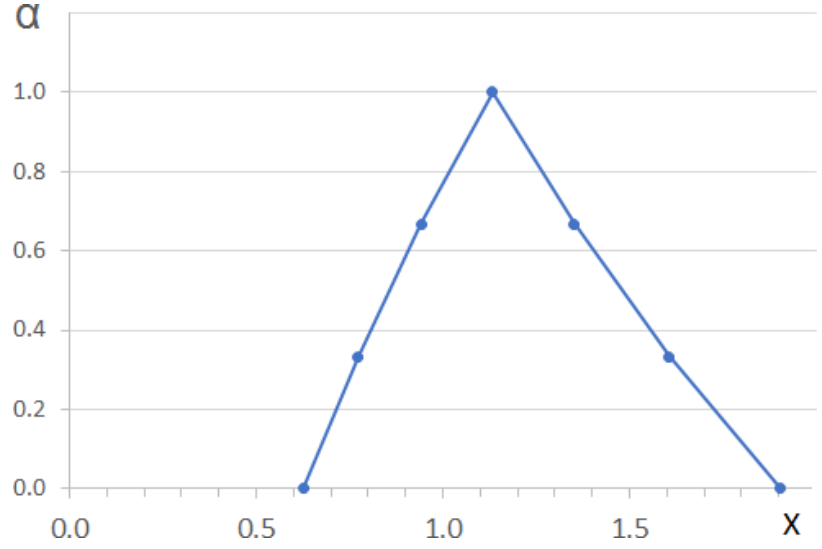

Figure 3. Membership function of $T_{\text {tech }}$

It is seen from the graph of the membership function, that the central value is shifted to the left, and the deviations from it are not quite symmetrical.

The proposed methods can be used when working with other economic indicators of a fuzzy nature to take into account the uncertainty associated with the fuzzy estimation of their components.

Consider the possibility of applying these methods on the example of estimating the fuzzy duration of a technological operation $T$, consisting of several elements, which duration is presented as fuzzy numbers.

The parameters for a fuzzy description of each operation element are calculated based on the statistical analysis of the observations on the duration of the operation elements at one workplace for one work shift (table 3). Using fuzzy numbers as the duration of each operation element, we can determine the fuzzy duration of the entire operation using some methods of performing arithmetic operations on fuzzy numbers (table 4). Observational data is considered stable because the coefficients of constancy $K_{s t}=\max / \min$ (used in labor rationing) do not exceed the allowable values for this type of production.

Table 3. Technological operation elements

\begin{tabular}{lc}
\hline Element description & Element name \\
\hline Take workpiece, install and fasten & $T_{1}$ \\
Switch on machine, withdraw tool & $T_{2}$ \\
Sharpen the part in one pass & $T_{3}$ \\
Withdraw tool, stop speed & $T_{4}$ \\
Unfasten, remove, set aside & $T_{5}$ \\
\hline
\end{tabular}

Both triangular and trapezoidal fuzzy numbers can be used to describe the duration of the technological operation elements. For trapezoidal numbers, we use the values of the boundaries of the entropy interval as the interval on which the membership function is equal to 1 . Four parameters for each description of the number are given in table 5 and table 6 .

Using the tools of programming language $\mathrm{R}$, we consistently find the sums of the fuzzy numbers described above for triangular as well as trapezoidal membership functions [16]. The results of fuzzy calculations we sum-
Table 4. Observations on the operation duration

\begin{tabular}{cccccc}
\hline \multirow{2}{*}{ Observation } & \multicolumn{5}{c}{ Duration of operation element, sec. } \\
\cline { 2 - 6 } & $T_{1}$ & $T_{2}$ & $T_{3}$ & $T_{4}$ & $T_{5}$ \\
\hline 1 & 9 & 3 & 103 & 4 & 5 \\
2 & 10 & 5 & 102 & 3 & 6 \\
3 & 7 & 4 & 115 & 5 & 7 \\
4 & 11 & 3 & 118 & 4 & 5 \\
5 & 14 & 4 & 121 & 6 & 9 \\
6 & 8 & 5 & 136 & 4 & 7 \\
7 & 12 & 7 & 139 & 6 & 6 \\
8 & 11 & 6 & 110 & 5 & 10 \\
9 & 13 & 4 & 128 & 4 & 8 \\
10 & 8 & 5 & 125 & 6 & 6 \\
$K_{s t}$ & 2 & 2.33 & 1.36 & 2 & 2 \\
\hline
\end{tabular}

Table 5. Fuzzy duration of technological operation elements (triangular fuzzy numbers)

\begin{tabular}{lllll}
\hline Element name & $a_{1}$ & $a_{2}$ & $a_{3}$ & $a_{4}$ \\
\hline$T_{1}$ & 7.0 & 10.47 & 10.47 & 14.0 \\
$T_{2}$ & 3.0 & 4.74 & 4.74 & 7.0 \\
$T_{3}$ & 102.0 & 121.61 & 121.61 & 139.0 \\
$T_{4}$ & 3.0 & 4.78 & 4.78 & 6.0 \\
$T_{5}$ & 5.0 & 7.05 & 7.05 & 10.0 \\
$T_{1}+T_{2}$ & 10.0 & 15.21 & 15.21 & 21.0 \\
$T_{3}+T_{4}$ & 105.0 & 126.39 & 126.39 & 145.0 \\
$\sum_{i=1}^{4} T_{i}$ & 115.00 & 141.6 & 141.6 & 166.0 \\
$\sum_{i=1}^{5} T_{i}$ & 120.00 & 148.65 & 148.65 & 176.0 \\
\hline
\end{tabular}

Table 6. Fuzzy duration of technological operation elements (trapezoidal fuzzy numbers)

\begin{tabular}{lllll}
\hline Element name & $a_{1}$ & $a_{2}$ & $a_{3}$ & $a_{4}$ \\
\hline$T_{1}$ & 7.0 & 8.89 & 12.03 & 14.0 \\
$T_{2}$ & 3.0 & 3.97 & 5.51 & 7.0 \\
$T_{3}$ & 102.0 & 113.97 & 129.21 & 139.0 \\
$T_{4}$ & 3.0 & 4.00 & 5.45 & 6.0 \\
$T_{5}$ & 5.0 & 5.97 & 8.14 & 10.0 \\
$T_{1}+T_{2}$ & 10.0 & 12.86 & 17.54 & 21.0 \\
$T_{3}+T_{4}$ & 105.0 & 117.97 & 134.66 & 145.0 \\
$\sum_{i=1}^{4} T_{i}$ & 115.0 & 130.83 & 152.2 & 166.0 \\
$\sum_{i=1}^{5} T_{i}$ & 120.0 & 137.88 & 159.25 & 176.0 \\
\hline
\end{tabular}

marize in table 5 and table 6 , and then provide a graphical representation of the resulting membership function for two mentioned types: triangular (figure 4) and trapezoidal (figure 5).

It is seen from the graphs of membership functions of the resulting indicators, that when performing the addition operation, the shape of the resulting membership function coincides with the shape of the membership function of terms, and the real interval of the fuzzy number, which determines the duration of the entire technological operation, does not depend on the membership function type.

As we can see, the carriers of fuzzy numbers are the same for both forms of fuzzy numbers, and the resulting membership functions allow us to make a conclusion about the fuzzy duration of the technological operation. This will focus the production system on the stabiliza- 


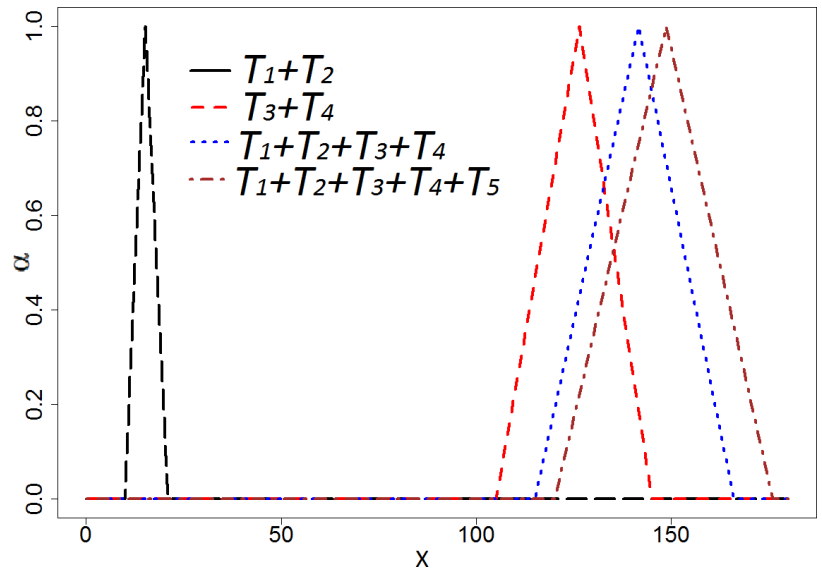

Figure 4. Membership functions of duration of technological operation and its elements (triangular fuzzy numbers)

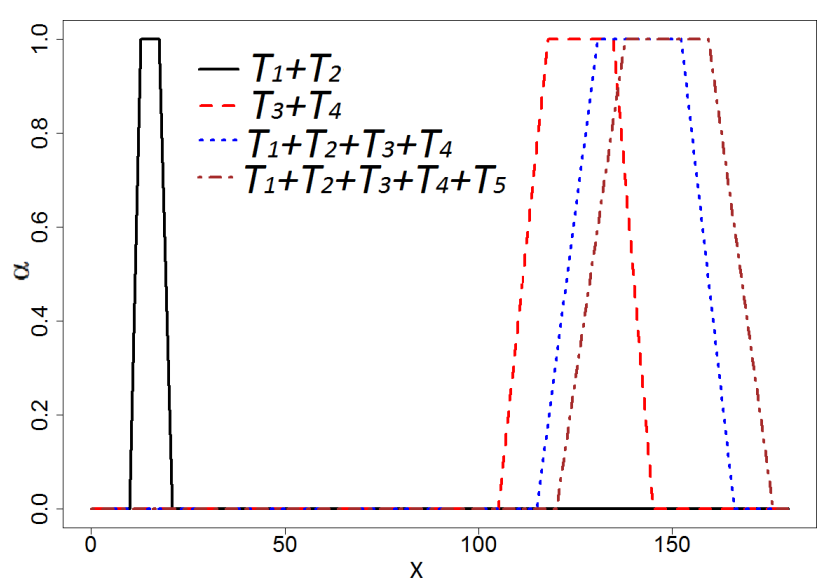

Figure 5. Membership functions of duration of technological operation and its elements (trapezoidal fuzzy numbers)

tion of labor processes by reducing the need for regulation within the fuzzy values of norms. In particular, such fuzzy estimators of the duration of different technological operations could be used in planning and forecasting the results of production processes on the enterprises. So, the decision-making systems can rely on more adequate and consistent information about labor and production processes, which allows determining the entire state of economic development on the enterprise.

\section{Results and discussion}

The stated approach to modeling uncertainty in economic indicators using fuzzy numbers provides considerable opportunities to obtain the most adequate estimates of these indicators using all available information on their structure and dynamics. Fuzzy arithmetic gives a powerful tool, which allows working with fuzzy economic indicators. We considered the appropriate theoretically sound calculation methods, and showed the examples of their use by modern computing tools.

In particular, the programming language $\mathrm{R}$, as freely available and open-source fuzzy system software, was used for automating arithmetic calculations using a fuzzy representation of economic indicators. We have shown that this approach is quite objective in terms of a formalized description of the existing uncertainty in any economic indicators, and as an example in the labor rationing. $\mathrm{R}$ is an ideal choice of language for such a toolbox for fuzzy calculations, as it is freely available and used by a wide variety of researchers from plenty of research fields [14].

\section{Conclusion}

In this paper, we have analyzed the state of development of the fuzzy arithmetic approach in economic problems in order to provide samples of application fuzzy arithmetic methods in estimating different economic indicators related to a particular branch (for example, labor rationing), and to focus on significant further developments.

Further research should improve the methodology of calculating fuzzy values of indicators used in other economic branches, and it will allow achieving a more adequate description of uncertainties in economic indicators, and to some extent simplify the decision-making processes in economic tasks. With this approach, decision-makers will be able to have more space to define important economic indicators and obtain more valuable information from the final results.

\section{References}

[1] L.A. Zadeh, Information and Control 8, 338 (1965)

[2] L. Zadeh, Information Sciences 8, 199 (1975)

[3] A.M.S. Ramasamy, Fuzzy arithmetic, in Proceedings of the National Seminar on Algebra and It's applications (Annamalainagar, 1999), pp. 213-223, https: //www.researchgate.net/ publication/279973774_FUZZY_ARITHMETIC

[4] L. Stefanini, L. Sorini, M.L. Guerra, in Handbook of Granular Computing (John Wiley \& Sons, Ltd, 2008), chap. 12, pp. 249-283, ISBN 9780470724163, https: //onlinelibrary.wiley.com/doi/abs/10. 1002/9780470724163. ch12

[5] L. Stefanini, New Tools in Fuzzy Arithmetic with Fuzzy Numbers, in Information Processing and Management of Uncertainty in Knowledge-Based Systems. Applications, edited by E. Hüllermeier, R. Kruse, F. Hoffmann (Springer Berlin Heidelberg, Berlin, Heidelberg, 2010), pp. 471-480, ISBN 9783-642-14058-7

[6] Y. Wang, Journal of Advanced Mathematics and Applications 3, 165 (2014)

[7] P. Dutta, H. Boruah, T. Ali, International Journal of Latest Trends in Computing 2, 99 (2011)

[8] S. Gao, Z. Zhang, C. Cao, Journal of Software 4, 331 (2009)

[9] D.A. Wismer (McGraw-Hill, New York, 1971), chap. 6. Distributed multilevel systems, p. 233-273 
[10] N. Gerami Seresht, A.R. Fayek, International Journal of Approximate Reasoning 106, 172 (2019)

[11] J.P. Mukeba, Journal of Fuzzy Set Valued Analysis 2019, 1 (2019)

[12] V. Balopoulos, Efficient Interval-Based Fuzzy Arithmetic, in 1st International Conference "From Scientific Computing to Computational Engineering”, Athens, Greece, 8-10 September 2004, edited by D.T. Tsahalis (Patras University Press, 2004), Vol. 2, pp. 498-505, https://www. researchgate. net/publication/236019730_Efficient_ Interval-Based_Fuzzy_Arithmetic

[13] L. Stefanini, L. Sorini, Fuzzy Arithmetic with Parametric LR Fuzzy Numbers, in Proceedings of the Joint 2009 International Fuzzy Systems Association World Congress and 2009 European Society of Fuzzy Logic and Technology Conference, Lisbon, Portugal, July 20-24, 2009, edited by J.P. Carvalho, D. Dubois, U. Kaymak, J.M. da Costa Sousa (2009), pp. 600605, http://www. eusflat.org/proceedings/ IFSA-EUSFLAT \_2009/pdf/tema \_0600.pdf

[14] C. Wagner, S. Miller, J.M. Garibaldi, A fuzzy toolbox for the $R$ programming language, in 2011 IEEE International Conference on Fuzzy Systems (FUZZ-
IEEE 2011) (2011), pp. 1185-1192

[15] J. Alcalá-Fdez, J.M. Alonso, IEEE Transactions on Fuzzy Systems 24, 40 (2016)

[16] M. Gagolewski, J. Caha, A Guide to the FuzzyNumbers Package for $R$ (2015), https://cran.r-project.org/web/ packages/FuzzyNumbers/vignettes/ FuzzyNumbersTutorial.pdf.

[17] B. de Bruyn, L. Fayet, V. Laborie, E3S Web of Conferences 7, 18002 (2016)

[18] Cédric Lesage, E.J.E.S.S. 15, 49 (2001)

[19] W.M. Wan Mohamad, T. Ahmad, N.A. Ab Karim, A. Ashaari, Mathematical Modelling and Analyses 23, 101 (2018)

[20] S.M. Lee, K.L. Lin, S. Gupta, Economic decision making using fuzzy numbers, in Proceedings of 2nd World Conference of POM (2003), Vol. 15, https://www. poms.org/archive/ conferences/Meeting2004/POMS_CDDel/ Browse\%20This\%20CD/PAPERS/002-0634 .pdf

[21] H.A. Sturges, Journal of the American Statistical Association 21, 65 (1926), https ://doi .org/10.1080/01621459.1926.10502161 